\title{
Expansion and characterization of tumor-infiltrating lymphocytes from human sarcoma
}

\author{
MacLean Hall ${ }^{*}$, John Mullinax¹, Erica Royster ${ }^{1}$, Angela Reagan¹, Ricardo Gonzalez', Jose Pimiento', \\ Shari Pilon-Thomas ${ }^{2}$, Amod Sarnaik ${ }^{1}$ \\ From 30th Annual Meeting and Associated Programs of the Society for Immunotherapy of Cancer (SITC 2015) \\ National Harbor, MD, USA. 4-8 November 2015
}

\section{Background}

Adoptive Cell Transfer (ACT) using Tumor Infiltrating Lymphocytes (TIL) has previously been shown at our institution and others to be an effective treatment for metastatic melanoma, resulting in a $38 \%$ response rate [1]. We applied this therapy to other solid tumors that have demonstrated a positive correlation between immune infiltrates and patient outcome [2]. Specifically in sarcoma, intra-tumoral CD4+ and CD8+ T cells have been detected, but their potential for ex vivo expansion is still relatively unexplored [3]. In this study, we investigated the feasibility of expanding TIL from surgically resected sarcoma specimens and analyzed the phenotype of these lymphocytes.

\section{Methods}

Four different subtypes of sarcoma were surgically resected from patients accrued under an IRB approved research protocol (MCC50064). A portion of the tumor specimens was digested and immediately phenotyped by flow cytometry. The remaining tumor was minced and plated as fragments for the isolation of TIL, which were expanded in vitro for six weeks using high dose IL-2. Eight separate TIL cultures were established and phenotyped by flow cytometry.

\section{Results}

Analysis of enzymatically digested human sarcoma specimens showed that $64 \%$ of lymphocytic infiltrates were CD3+ cells. TIL were isolated from fragments of each of the four sarcoma specimens as eight individual cultures and propagated in vitro, with TIL observed in 59 out of 84 (70\%) fragments. Of the expanded CD3+ TIL, on average $39 \%$ were CD8+ T cells that expressed both the co-stimulatory molecule 4-1BB (33\%) and inhibitory PD-1 (41\%) by flow cytometry.

\section{Conclusions}

Human sarcoma specimens yield CD3+ CD8+ TIL which can be expanded in vitro, supporting further investigation into the feasibility of adoptive cell transfer as a therapy for these patients. Efforts are currently focused on the scalability of this process and the functional capacity of these TIL. Additionally, the expression of $4-1 B B$ and PD-1 on a substantial of CD3+ CD8+ TIL demonstrates the opportunity to modulate these pathways to improve both yield and function, another endeavor we are presently investigating.

\section{Authors' details}

${ }^{1} \mathrm{H}$. Lee Moffitt Cancer Center, Tampa, FL, USA. ${ }^{2} \mathrm{H}$. Lee Moffitt Cancer Center \& Research Institute, Tampa, FL, USA.

\section{Published: 4 November 2015}

\section{References}

1. Pilon-Thomas S, Kuhn L, Ellwanger S, Janssen W, Royster E, Marzban S, et al: Efficacy of adoptive cell transfer of tumor-infiltrating lymphocytes after lymphopenia induction for metastatic melanoma. J Immunother 35(8):615-620.

2. Turcotte S, Gros A, Hogan K, Tran K, Hinrichs CS, Wunderlich JR, et al: Phenotype and function of $T$ cells infiltrating visceral metastases from gastrointestinal cancers and melanoma: implications for adoptive cell transfer therapy. J Immunol 191(5):2217-2225.

3. Tseng W, Malu S, Zhang M, Chen J, Sim GC, Wei W, et al: Analysis of the Intratumoral Adaptive Immune Response in Well Differentiated and Dedifferentiated Retroperitoneal Liposarcoma. Sarcoma 2015:547460.

doi:10.1186/2051-1426-3-S2-P19

Cite this article as: Hall et al:: Expansion and characterization of tumorinfiltrating lymphocytes from human sarcoma. Journal for ImmunoTherapy of Cancer 2015 3(Suppl 2):P19.

${ }^{1} \mathrm{H}$. Lee Moffitt Cancer Center, Tampa, FL, USA

Full list of author information is available at the end of the article 